\title{
HF-PUMP-INDUCED PARAMETRIC INSTABILITIES IN THE AURORAL E-REGION
}

\author{
M. T. Rietveld ${ }^{1,7}$, B. Isham ${ }^{2,7}$, T. Grydeland ${ }^{3}$, C. La Hoz ${ }^{3}$,

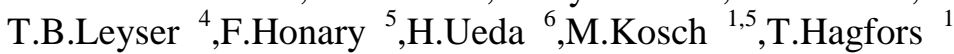 \\ ${ }^{1}$ Max-Planck-Institut für Aeronomie, Germany \\ ${ }^{2}$ Interamerican University, Bayamón, Puerto Rico, USA \\ ${ }^{3}$ Auroral Observatory, Troms $\phi$, Norway \\ ${ }^{4}$ Swedish Institute of Space Physics, Uppsala, Sweden \\ ${ }^{5}$ Lancaster University, $U K$ \\ ${ }^{6}$ Chiba University, Japan \\ ${ }^{7}$ also at EISCAT Scientific Association, Ramfjordmoen, Norway
}

\begin{abstract}
In November 1999 the EISCAT high-power, high-frequency (HF) facility located near Troms $\varnothing$, Norway, was used to create artificial plasma turbulence in the ionosphere. During the experiment the EISCAT $224 \mathrm{MHz}$ radar and sometimes the $931 \mathrm{MHz}$ radar were used to obtain measurements of incoherent scatter ion and plasma lines, and artificially enhanced spectra of E-region plasma waves were measured for the first time at auroral latitudes with both radars. During periods with suitable peak E-region electron density, Z-mode propagation of the HF pump wave to the topside E-region occurred, and topside instability-enhanced plasma waves were observed. In addition to HF-pump-induced effects, an unusual F-region echo was seen in both the ion and plasma line channels, which appears to be due to an auroral arc intersecting the radar beam.
\end{abstract}

\section{INTRODUCTION}

At the high latitude European Incoherent Scatter (EISCAT) site near Troms $\varnothing$, Norway, high frequency (HF) experiments involving the excitation of plasma instabilities using O-mode transmissions have been done mainly during the daytime. The reasons are a frequent lack of high enough electron density at night, especially near solar minimum, and the desire to avoid the ionospheric disturbances caused by auroral activity. Recently, however, such experiments have been attempted at night in order to study the artificial airglow and large electron temperature enhancements which may be created (e.g. Leyser et al., 2000). During a campaign in November 1999, a new data-taking program was implemented on the EISCAT very high frequency (VHF, $224 \mathrm{MHz}$ ) and ultra high frequency (UHF, $931 \mathrm{MHz}$ ) incoherent scatter radars which extended the scope of the observations to include ion and plasma lines from the E-region and the topside ionosphere with reasonable altitude resolution. E-region HFenhanced plasma and ion lines were observed. These observations are the first from high latitudes.

Plasma waves excited in a sporadic-E layer by powerful HF wave injection were first reported using the $430 \mathrm{MHz}$ incoherent scatter radar at Arecibo by Gordon and Carlson (1976). Further results, in both normal daytime and sporadic E-layers, were obtained by Djuth (1984) and their temporal evolution was studied by Djuth and Gonzales (1988). All the E-region plasma line observations at Arecibo showed narrow spectral features at $430 \mathrm{MHz} \pm f_{\mathrm{HF}}$ where $f_{\mathrm{HF}}$ is the HF pump frequency. At the EISCAT facilities, enhanced E-region ion lines were observed with the UHF incoherent scatter radar by Schlegel et al. (1987), but spectra were not reported and there were no plasma line measurements.

Based on the Arecibo results, two candidate interaction mechanisms have been postulated. One is the modulational instability, also known as the oscillating two-stream instability or the purely-growing parametric decay instability, where the HF wave decays into two oppositely directed Langmuir waves having frequencies 
equal to $f_{\mathrm{HF}}$ and two ion acoustic waves shifted to zero frequency (Muldrew, 1978; Nishikawa, 1968). The other mechanism is direct conversion of the HF pump into Langmuir waves by ionospheric irregularities (Djuth, 1984).

\section{TECHNIQUE}

\section{HF modulation}

On 11 Nov. 1999 the EISCAT HF facility was used to transmit an effective radiated power of $240 \mathrm{MW}$ at $4.04 \mathrm{MHz}$ with a $10 \mathrm{~s}$ on, $10 \mathrm{~s}$ off square wave modulation sequence. Before 18:27 UT only $\mathrm{O}$ mode was transmitted, after 18:27 UT the polarization alternated between X mode during the odd minutes and $\mathrm{O}$ mode during even minutes. The HF beam was directed vertically.

\section{Radar data taking program}

The data presented here were taken with programs created using software developed by T. Grydeland. The core of the experiment is a new correlator program, called EPLA2 (E-region plasma line experiment number 2) built from general purpose GEN (for the long pulse (Turunen, 1985, 1986)) and G2 (for the alternating code (Wannberg, 1993)) system subroutines. With the exception of the radar frequencies, the same program was run on both the VHF and UHF radars. The radar program combines long pulse, power profile (short pulse) and alternating code modulations, with three receiver channels receiving the power profile and the alternating code and one channel receiving the long pulse. This means that both the ion line and two different plasma line offsets can be monitored simultaneously. The alternating code channels (Lehtinen, 1986; Lehtinen and Häggström, 1987) allowed us to record high-resolution spectra of artificially induced ion and plasma lines in the E and F-regions. The long pulse was used to record ion line spectral data for analysis of background parameters.

For the observations presented in this paper, we used 20 bits of a 32-bit alternating code with a baud length of $25 \mu \mathrm{s}$, resulting in a range resolution of $3.75 \mathrm{~km}$, covering ranges from 90 to $311 \mathrm{~km}$. The lag resolution is $25 \mu \mathrm{s}$, and lags from 25 to $475 \mu \mathrm{s}$ are covered, resulting in a frequency resolution of nearly $2 \mathrm{kHz}$. This is sufficient for observation of the ion spectrum at these altitudes. The power profile used the same range resolution as the alternating code, but with the range extended to $475 \mathrm{~km}$. The long pulse transmission was $420 \mu \mathrm{s}$ in duration and was used to record data in the ranges from 180 to $780 \mathrm{~km}$. The power profile short pulse and alternating code were transmitted 422 and $6,394 \mu$ s after the start of the long pulse, respectively. The VHF transmission frequencies were 223.6 (222.4) $\mathrm{MHz}$ (long pulse) and 222.6 (223.4) $\mathrm{MHz}$ (power profile and alternating code pairs) for set 1 (set 2). The UHF transmission frequencies were 930.5 (927.5) $\mathrm{MHz}$ (long pulse) and 928.0 (930.0) $\mathrm{MHz}$ (power profile and alternating code pairs) for set 1 (set 2). The pulse repetition period for a single pair of transmission sets was 38,506 $\mu \mathrm{s} ; 256$ repetitions of the transmission pairs (four complete alternating code cycles) were made during each 10 -s integration period.

The VHF radar antenna was pointed vertically while the UHF antenna was pointed at $81^{\circ}$ elevation towards the south; the UHF radar ran only part of the time and suffered from low transmitter power and a noisy receiver. The magnetic dip angle at Troms $\varnothing$ is $13^{\circ}$.

\section{Supporting observations}

A co-located digital HF sounder (ionosonde) made fixed and swept frequency soundings. Other diagnostics included an all-sky airglow imaging system, stimulated electromagnetic emission (SEE) spectral measurements, and HF and VHF coherent scatter radar observations. Some of these supporting data will be published elsewhere.

\section{RESULTS}

\section{VHF and UHF spectra}

Here we present EISCAT VHF and UHF radar results for 11 Nov. 1999. Figure 1 shows an overview of the intensity of the VHF echoes seen in the three power profile channels during one hour on 11 Nov. 1999. The bottom panel shows the ion line channel. The gray regions between 100 and $200 \mathrm{~km}$ are from the auroral E-region. The black dots at the lower edge of the gray are echoes from enhanced backscatter due to HF pumping. The center panel shows the downshifted plasma line (DPL) at $-4.04 \mathrm{MHz}$ and the top panel shows the upshifted plasma line (UPL) at +4.04 MHz. The HF-pump-enhanced signals are stronger and therefore seen more clearly in these channels. Around 18:17 and after 18:36 UT a number of enhanced signals come from F-region heights, 200 to $350 \mathrm{~km}$, during periods when the E-region becomes underdense. After 18:50 UT the E-region echoes appear on both the bottom and topside E-region in all three channels. HF-induced enhancements are seen only during O- 


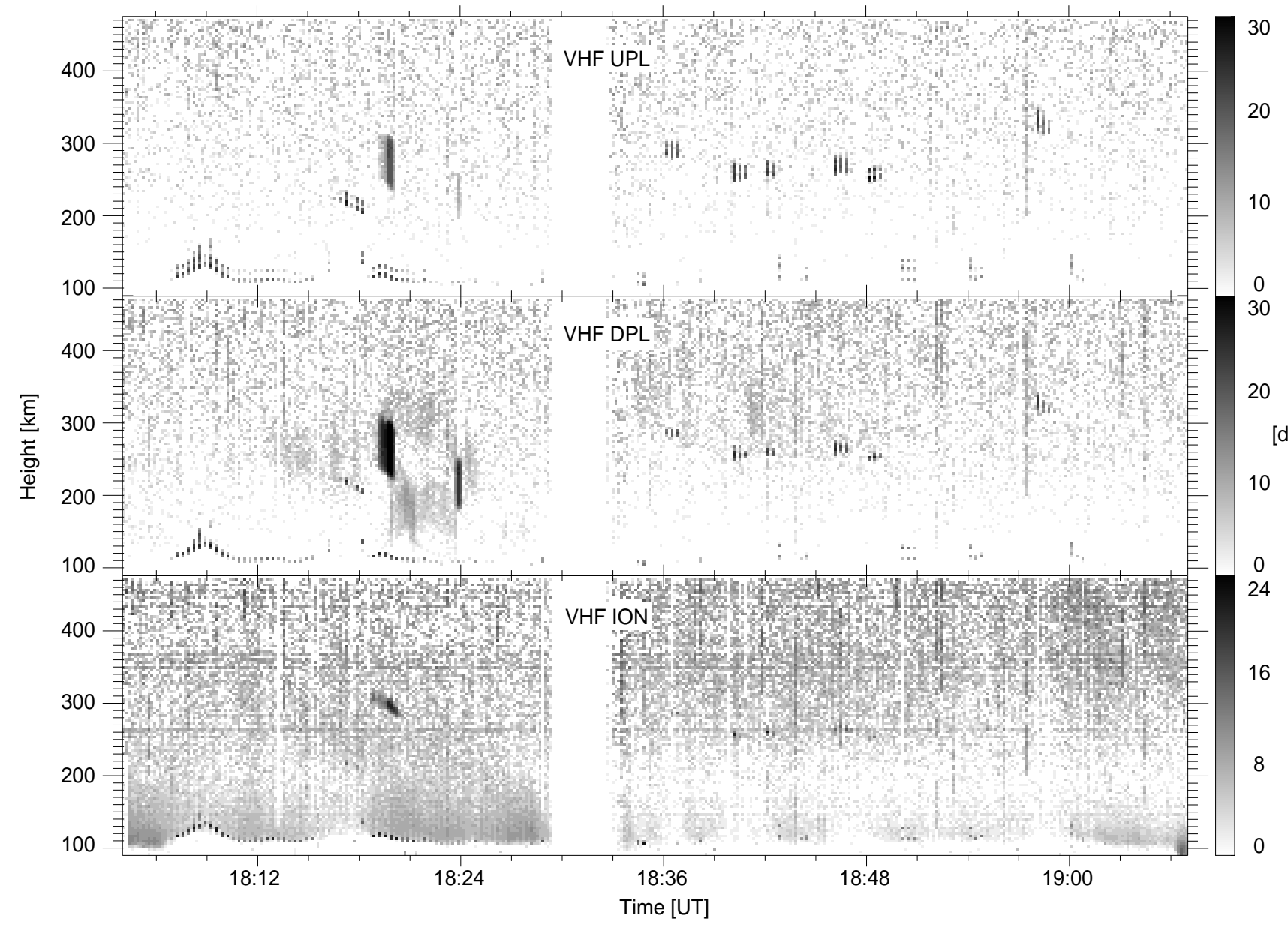

Fig. 1. Overview of the backscatter intensity in the three VHF power profile channels on 11 Nov. 1999. The bottom panel shows the ion line, while the upper panels show the downshifted plasma line (DPL) and upshifted plasma line (UPL), which are offset by - and $+4.03 \mathrm{MHz}$ from the radar frequency, respectively. All three channels have a linear $\pm 25-\mathrm{kHz}$ bandwidth.

mode pumping. The intense F-region echoes between 18:19 and 18:24 UT are discussed later. In the UPL channel (top panel) an artefact of the experiment causes the echoes to be repeated about $15 \mathrm{~km}$ above the real echoes. When echoes appear from both the bottom and topside E-region this technical fault makes it appear as if there are three scattering regions.

Figures 2 and 3 show spectra from two 10-s intervals where HF-enhanced ion and plasma lines are excited in the E-region. In both figures the VHF plasma lines show a strong line offset from the HF frequency towards the radar frequency by the ion-acoustic frequency (about $1.5 \mathrm{kHz}$ ), which is not quite resolved. This feature, known as the decay line, is produced by the parametric decay instability (e.g. Hanssen et al., 1992). The UHF plasma line spectra in Figure 2 show both a decay line and a line at the HF pump frequency, which appears to be the purely growing line produced by the modulational instability, with the former being stronger. In the UHF spectra in Figure 3 only the decay line is present. The UHF ion line always shows strongly enhanced ion acoustic shoulders and a zero-frequency component, features produced by the parametric decay and modulational instabilities, respectively (Sprague and Fejer, 1995). The VHF UPL spectra show a first cascade, a feature offset towards the radar frequency (inshifted) from the decay line by twice the ion acoustic frequency or about $3 \mathrm{kHz}$. There is also a line outshifted from the decay line by about $3 \mathrm{kHz}$, which may be the image decay line, also known as the antiStokes line (Djuth, 1984; Stubbe et al., 1992; DuBois and Goldman, 1967; Goldman et al., 1995). The height difference of $7 \mathrm{~km}$ between UHF and VHF excitation in Figure 3 must be attributed in large measure to a spatially and temporally varying E-region, since the radars are pointing to regions $18 \mathrm{~km}$ apart. For example, twenty seconds later the excitation observed by the VHF radar had dropped from about $125 \mathrm{~km}$ to approximately $119 \mathrm{~km}$ altitude, while the UHF excitation height dropped from $119 \mathrm{~km}$ to $115 \mathrm{~km}$. Furthermore, although the height of the VHF return was usually above that of the UHF signal by more than a kilometer, the VHF height was occasionally lower, although by a kilometer or less. Keeping this probably largely natural variability in mind, the height difference may be argued to be consistent with the relative difference in the altitudes where parametric 

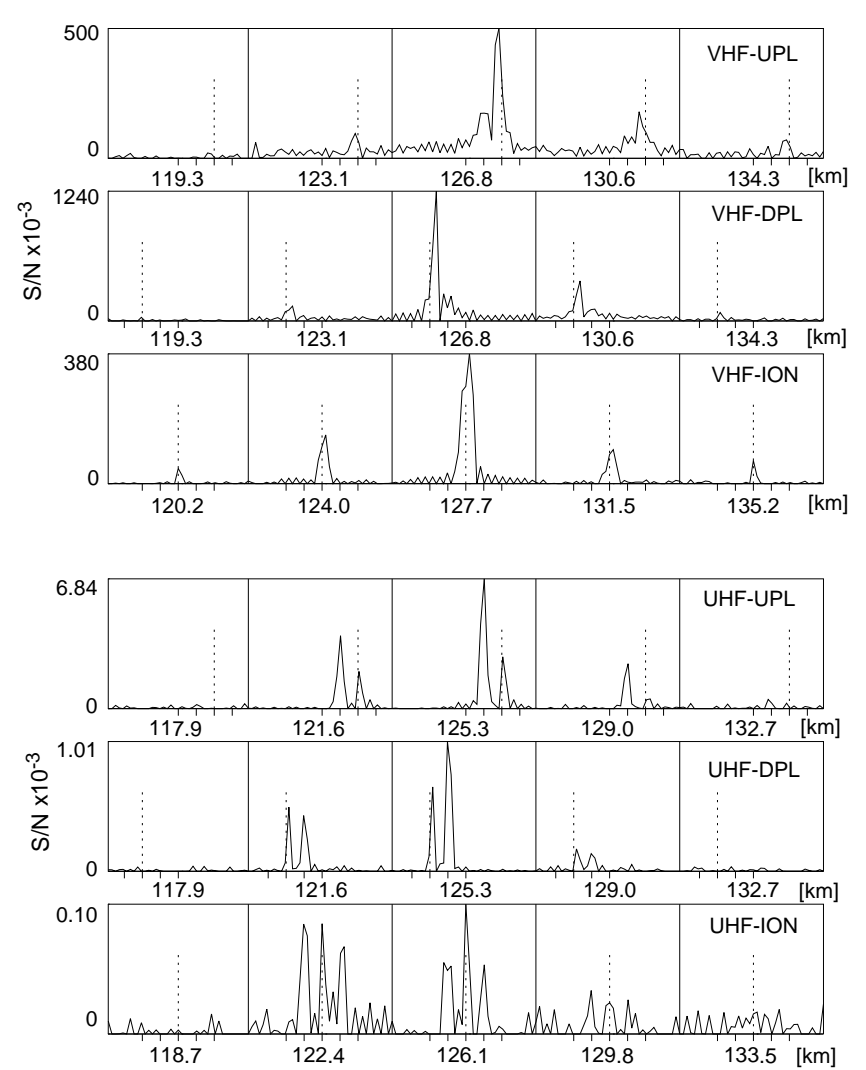

Fig. 2. VHF and UHF spectra from HF-enhanced ion and plasma lines in the E-region at 18:08:00 to 18:08:10 UT. The tic marks are at $5-\mathrm{kHz}$ intervals. The numbers below each spectrum show the altitude in $\mathrm{km}$. The dotted lines in the UPL and DPL spectra indicate the HF pump frequency. The VHF plasma line spectra show mainly the decay line whereas the UHF spectra show both the decay and the modulational instability lines. The ion acoustic frequency is about $5 \mathrm{kHz}$ and $1.2 \mathrm{kHz}$ at the UHF and VHF radar $\mathrm{k}$ vectors, respectively.
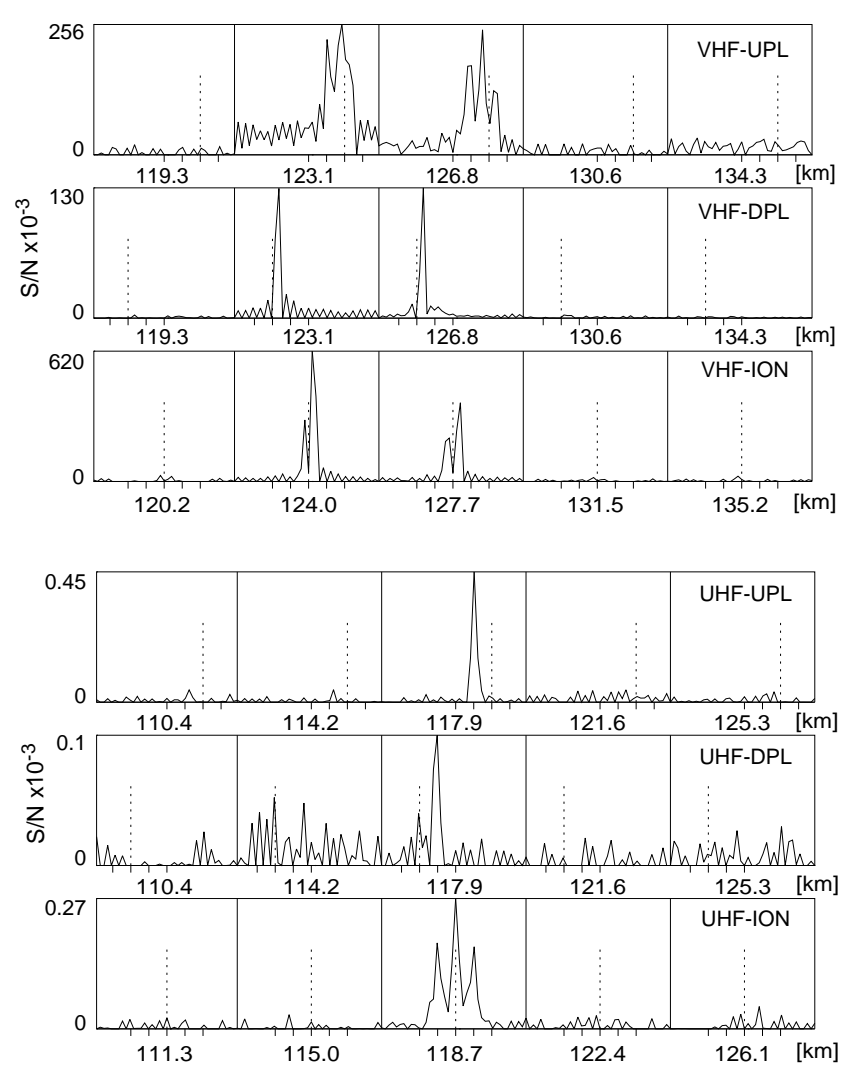

Fig. 3. VHF and UHF spectra similar to those in Figure 2 but at 18:09:20 to 18:09:30 UT. Here the UHF plasma line shows only the decay line, about $5 \mathrm{kHz}$ below the HF pump frequency.

instabilities are expected to be seen at the respective radar wavelengths, although the difference is expected to be less than $1 \mathrm{~km}$ in the E-region.

\section{Topside and bottomside E-region spectra}

Figure 4 shows VHF spectra from forty minutes later, when there were echoes from both the bottom and topside of the E-region (the UHF radar was off after 18:30 UT). The topside E-region enhancements are likely due to conversion of the HF pump wave from O to Z mode at the bottomside critical height, $\mathrm{Z}$ mode propagation through the E-region peak, and excitation of instabilities at the topside critical height by the Z-mode wave (Mishin et al., 1997; Isham et al., 1999). At this time the ionograms show a peak E-region frequency of $4.5 \mathrm{MHz}$, about $200 \mathrm{kHz}$ below the Z-mode critical frequency.

The topside spectra in Figure 4 show clear decay lines, little if any trace of a cascade line, and no indication of a modulational instability line. The spectra are stronger on the topside than on the bottomside, which may be due to greater collisional damping at the lower bottomside altitude, but may also be a clue to the efficiency of the $\mathrm{O}$ to Z-mode coupling process. On the other hand, collisional damping at the lower bottomside altitude is likely a principle cause, and returns recorded at the same altitude around 18:12 UT, when there was no topside enhancement, have similar weak intensity. In addition, the relative strength of the bottomside vs. topside ion line varied with time. This type of variability, likely a result of disturbances due to auroral activity and created by the $50 \% \mathrm{HF}$ transmission duty cycle, makes it difficult to draw firm conclusions. However, the bottomside spectra appear to consist of a broadened decay line and an ion line in which the two shoulders have merged into a single feature; which is consistent with greater collisional damping at those altitudes.

\section{Unusual F-region echoes}

Unusual and interesting echoes from the F-region were observed between 18:19 and 18:24 UT (Fig. 1). At 18:19 an enhanced ion line descends in the power profile from 310 to $280 \mathrm{~km}$ and a large region of strong backscatter is seen in both plasma line power profile channels. These strong echoes are the result of the long pulse transmissions encountering a relatively thin region of enhanced scattering, with the backscatter being received in 
the power profile channels. This can be deduced from the range extent of the echo (the long pulse is $420 \mu \mathrm{s}$ or $63 \mathrm{~km}$ long), and from the timing of the long pulse transmissions, which end $2 \mu$ sefore the short pulse, so that the upper edge of the echo in the plasma line channels corresponds closely to the altitude of the thin ion line trace. Because the data in each time-integrated power profile channel is obtained from two physical channels tuned to different frequencies, the DPL power profile channel is sensitive to long pulse plasma lines centered on $-5.03 \mathrm{MHz}(=-4.03-223.4+222.4)$ and $-3.03 \mathrm{MHz} \quad(=-4.03-222.6+223.6)$ and the UPL power profile channel to $+3.03 \mathrm{MHz}$ and $+5.03 \mathrm{MHz}$. That the echoes are in fact 5.03 and not $3.03 \mathrm{MHz}$

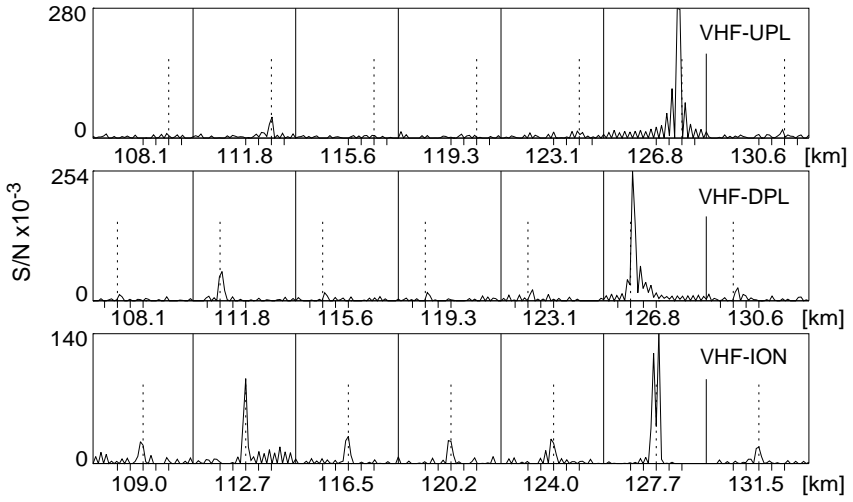

Fig. 4. VHF spectra from top and bottomside E-region echoes from 18:50:00 to 18:50:10 UT. plasma lines is deduced from the previous HF-enhanced plasma lines at $4.04 \mathrm{MHz}$ together with electron density profiles derived from the ion line. The power-profile channels were tuned for a $4.03 \mathrm{MHz}$ offset rather than 4.04 MHz. Thin traces in the UPL and DPL channels corresponding to the $\pm 4.04 \mathrm{MHz}$ plasma lines may also be present, but if so they are probably masked by the strong and longer-lasting long pulse echo. The ion line power profile, which similarly measures plasma lines at $\pm 1 \mathrm{MHz}$ from the two long pulses, does not show corresponding enhancements. The long pulse ion line spectrum (not shown) does show an enhancement at the correct height.

A weaker echo is seen at 18:24 UT at a lower height. It is strongest in the DPL but still strong in the UPL, but no corresponding ion line was detected. The echoes do not correlate with the HF modulation, but occur at the start and end of a period of naturally enhanced electron temperature (data not shown). At the same time there was auroral precipitation present which caused the E layer to be strongly enhanced. The echoes appear to come from a narrow region of natural plasma instability, probably related to an auroral arc intersecting the vertical radar beam. Similar examples, again without corresponding ion line signals, are seen at 19:16 UT and on the next day (12 Nov.) at 18:12 UT. The digital all-sky imager, situated at Skibotn about $50 \mathrm{~km}$ to the east, showed the presence of aurora on both days when these echoes were observed, although cloudy conditions made it impossible to determine the exact locations of the emissions. Enhanced ion-acoustic echoes have been seen relatively often at VHF (Rietveld et al., 1996; Collis et al., 1991) and less frequently at UHF.

There are also weaker dark traces of roughly the same altitude extent at other times and heights which may be $3 \mathrm{MHz}$ plasma lines. These unusual F-region echoes will be studied in greater detail in a separate publication.

\section{DISCUSSION}

The observation that E-region incoherent scatter spectra show features attributable to the parametric decay (UHF and VHF) and modulational (UHF only) instabilities is reminiscent of F-region results (e.g. Stubbe et al., 1992; Rietveld et al., 2000). This suggests that HF-pump-induced Langmuir turbulence is occurring in the Eregion as it does in the F-region, with the distinction that there are fewer cascade lines and that no broad (cavitating turbulence) spectra are visible. However, the $3.75-\mathrm{km}$ height resolution of the experiment was not good enough to resolve the features of cavitating turbulence. The main relevant differences between the $\mathrm{E}$ and $\mathrm{F}$ regions are (i) a higher frequency of collisions between electrons and heavy particles, and (ii) a larger density gradient. The power profiles indicate a plasma frequency gradient, $d f_{p} / d z$, below the bottomside E-region excitations of approximately $200-400 \mathrm{kHz} / \mathrm{km}$. Using the relation $d f_{p} / d z=f_{p} /(2 H)$ this corresponds to a scale height, $H$, of $5-10 \mathrm{~km}$. Sporadic $\mathrm{E}$ has $H \approx 0.2-1 \mathrm{~km}$ or less (Djuth 1984) and in the F-region $H$ may be between and 30 and $200 \mathrm{~km}$. Goldman et al., (1995) have made a theoretical study of Langmuir turbulence in a collisional regime and Goldman et al., (1997) applied the theory to a steep density gradient similar to ours but in the Fregion.

This observation suggests that instabilities, rather than direct conversion, are also responsible for the enhancements seen at Arecibo. Our results differ from those at Arecibo in that our plasma line spectra have features offset from the HF frequency. It would be useful to apply recent models of Langmuir turbulence to Eregion conditions, similar to Goldman et al., (1995; 1997). The results may be different because the geomagnetic field is much more closely aligned to the radar pointing direction at EISCAT than at Arecibo.

The geomagnetic field strongly affects other aspects of the observations as well. $\mathrm{Z}$ mode propagation is only possible since the radar and HF beams are closely aligned to the geomagnetic field. Furthermore, during the 
observations the UHF antenna was directed to $81^{\circ}$ south elevation, outside the Spitze region which extends only to $84^{\circ}$ south. So the UHF was observing HF rays which were refracted before they reached the critical level. The steep gradients in the E-region however ensure that the turnaround point is close to critical, and close to the radar matching height, where, in a smooth background plasma, parametric instabilities are expected to be observed.

The measured electron density profile might be accurate enough to compute the Airy swelling factor, and hence the pump field strength to compare with instability thresholds. Possible differences in the gradients on the bottom and topside may turn out to produce significantly different field strengths in those two regions, thus possibly affecting the relative strengths of the enhancements. D-region absorption will work to reduce the pump power, and can be estimated from the ionogram data. We know that the absorption was relatively low, as many of the ionograms show two or more hops by the HF wave. The geomagnetic activity and the 10-s-on, 10-s-off pump duty cycle, however, undoubtedly produced small scale plasma density irregularities, thus adding a random component to the electron density profile, the HF ray paths, and the strengths of the observed lines. These density irregularities could also increase the efficiency of the $\mathrm{O}$ to $\mathrm{Z}$ mode conversion.

\section{ACKNOWLEDGEMENTS}

EISCAT is supported by the research councils of Finland (SA), France (CNRS), Germany (MPG), Japan (NIPR), Norway (NFR), Sweden (NFR) and the United Kingdom (PPARC). T.G. thanks the EISCAT staff at Troms $\emptyset$ for their assistance in conducting the radar experiment. B.I. gratefully acknowledges support from U.S. National Science Foundation Ionospheric Interactions Initiative grant ATM-9818280, jointly sponsored by the High Frequency Auroral Active Research Program. T.B.L. gratefully acknowledges support from the Swedish Natural Science Research Council.

\section{REFERENCES}

Collis, P. N., I. Häggström, K. Kaila and M. T. Rietveld, EISCAT radar observations of enhanced incoherent scatter spectra; their relation to red aurora and field-aligned currents, Geophys. Res. Lett., 18, 1031-1034, 1991.

Djuth, F. T., HF-enhanced plasma lines in the lower ionosphere, Radio Sci., 19, 1, 383-394, 1984.

Djuth, F. T. and C. A. Gonzales, Temporal evolution of the HF-enhanced plasma line in sporadic E, J. Geophys. Res, 93, $196,1988$.

DuBois, D. F. and M. V. Goldman, Theory of four-plasmon parametric excitation and comparison with experiment, Phys. Rev. Lett., 19, 1105-1110, 1967.

Goldman, M. V., D. L. Newman, D. Russell, D. F. DuBois, H. Rose, R. P. Drake and A. M. Rubenchik, Collisional regimes of radiation-driven Langmuir turbulence, Phys. Plasmas 2, 1947-1960, 1995.

Goldman, M. V., D. L. Newman, R. P. Drake and B. B. Afeyan, Theory of convective saturation of Langmuir waves during ionospheric modification of a barium cloud, J. Atmos. Sol. Terr. Phy 59, 2335-2350, 1997.

Gordon, W. E. and H. C. Carlson, The excitation of plasma lines in blanketing sporadic E, J. Geophys. Res., 81, $4016,1976$.

Hanssen, A., E. Mjølhus and D. F. DuBois and H. A. Rose, Numerical test of the weak turbulence approximation to ionospheric Langmuir turbulence, J. Geophys. Res, 97, 12073-12091, 1992.

Isham, B., M. T. Rietveld, T. Hagfors, C. La Hoz, E. Mishin, W. Kofman, T. B. Leyser and A. P. van Eyken, Aspect angle dependence of HF enhanced incoherent backscatter, Adv. Space Res., 24, 1003-1006, 1999.

Lehtinen, M. S., Statistical theory of incoherent scatter measurements, Technical Report 86/45, EISCAT Scientific Association, Kiruna, Sweden, Ph.D. thesis, University of Helsinki, Helsinki, Finland, 1986.

Lehtinen, M. and I. Häggström, A new modulation principle for incoherent scatter measurements, Radio Sci, 22 , 4, $625,1987$.

Leyser, T. B., B. Gustavsson, B. U. E. Brändström, Å. Steen, F. Honary et al., Simultaneous measurements of high-frequency pump-enhanced airglow and ionospheric temperatures at auroral latitudes, Adv. Polar Upper Atmos. Res., 14, 1-11, 2000.

Mishin, E., T. Hagfors and W. Kofman, On the origin of outshifted plasma lines during HF modification experiments, $J$. Geophys. Res., 102, A12, 27265-27269, 1997.

Muldrew, D. B., Langmuir wave propagation and the enhanced plasma line in sporadic E, J. Geophys. Res., 83A, 5207, 1978.

Nishikawa, K., Parametric excitation of coupled waves, II parametric plasmon-photon interaction, J. Phys. Soc. Jpn., 24, $1152,1968$.

Rietveld, M. T., P. N. Collis, A. P. van Eyken and U. P. Løvhaug, Coherent echoes during EISCAT UHF Common Programmes, J. Atmos. Terr. Phys., 58, 161-174, 1996.

Rietveld, M. T., B. Isham, H. Kohl, C. La Hoz and T. Hagfors, Measurements of HF enhanced plasma and ion lines at EISCAT with high altitude resolution, J. Geophys. Res., 105A, 7429-7439, 2000.

Schlegel, K., M. Rietveld and A. Maul, A modification event of the auroral E region as studied with EISCAT and other diagnostics, Radio Sci., 22, 6, 1063-1072, 1987.

Sprague, R. A. and J. A. Fejer, Simultaneous excitation of parametric decay cascades and of the oscillating two-stream instability in one-dimensional numerical simulations based on Zakharov's equations, J. Geophys. Res. 100, $23959,1995$.

Stubbe, P., H. Kohl and M. Rietveld, Langmuir turbulence and ionospheric modification, J. Geophys. Res., 97A, 6285, 1992.

Turunen, T., The GEN-system for the EISCAT incoherent scatter radars, Tech. Report 85/44, EISCAT, Kiruna, Sweden, 1985. Turunen, T., GEN-system, A new experimental philosophy for the EISCAT radar, J. Atmos. Terr. Phys., 48, 9-10, 777, 1986.

Wannberg, G, The G2-system and general purpose alternating code experiments for EISCAT, J. Atmos. Terr. Phys, 55, 4/5, 543, 1993 